\title{
Viability of first and second generation hybrids of Drosophila virilis and Drosophila lummei
}

\author{
Jaakko Lumme and \\ Erja Heikkinen
}

Department of Genetics, University of Oulu, Linnanmaa, SF 90570 Oulu, Finland

$F_{1}$ hybrids of $D$. virilis and $D$. lummei survived significantly worse than the parents. When $D$. virilis was the mother, larger proportion of hybrids failed to emerge from pupal case, but overall survival rate of reciprocals was about the same. Viability of different heterospecific combinations of sex chromosomes and autosomes was studied in backcrosses. All the sex chromosomes survived well on the background of all-heterozygous autosomes. Hemizygous $X$ was reciprocally subvital with homozygous alien autosomes. The reduced viability was male sex limited. The X-linked factor causing this effect was localized in the area of the large, phylogenetically ancient double inversion In(1) $a+b$. All four large autosomes were heterotic in backcross hybrids, more strongly in females. Five out of six possible first degree interactions between the autosomes were negative, indicating incompatibility between heterospecific autosomes. This effect was stronger in females. The genetic basis for hybrid subvitality is sex-specific.

\section{INTRODUCTION}

Species hybrids are often less viable than pure species. As a metaphor, this is said to be due to the breakdown of co-adapted genetic system in hybridization. Subvitality of hybrids has been under genetic analysis in very few cases, much more seldom than hybrid sterility (Coyne and Orr, $1989 a, 1989 b)$. An important exception is the case of Drosophila melanogaster and its siblings. Among them, the viability of hybrids can be rescued by some mutants. The gene $L h r$ (Lethal hybrid rescue) was detected by Watanable (1979) in Drosophila simulans. Another mutant, $\mathrm{Hmr}$ (Hybrid male rescue) was found by Hutter and Ashburner (1987) in Drosophila melanogaster. The results of the further analysis are very important with respect to the understanding of the mechanisms of speciation in general (Hutter et al., 1990). In the species group observed in this paper, Patterson and Griffen (1944) studied in detail the inviability of female progeny from the cross of $D$. montana females to D. texana males. A gene within echinus-crossveinless interval of the texana $\mathrm{X}$ chromosome was lethal in combination with the

We dedicate this paper to Academician Esko Suomalainen, on the occasion of his 80th birthday. montana egg "protoplasm". Several other cases of hybrid lethality described in older literature were reviewed in Patterson and Stone (1952).

As a part of our investigations on the genetic differentiation of two allopatric sibling species Drosophila virilis and Drosophila lummei, we present here an analysis of the viability of different heterospecific combinations of sex chromosomes, and autosomes.

The model of speciation applicable to the present pair of taxa is through geographic isolation. Throckmorton (1982) presented the still valid phylogenic and biogeographical scheme for their separation. The geographical isolation continues: D. lummei shows a palaearctic boreal distribution, with suitable adaptations like better cold resistance (Heino and Lumme, 1989) and photoperiodic diapause (Lumme and Keranen, 1978). D. virilis has a more southern holarctic distribution, and it comes to the areas common with $D$. lummei only as aided by man.

The isolation of the taxa in question, expressed as subvitality of different heterospecific genotypes is rather weak. This fact was well known to us before beginning. The idea of studying weak isolation can be founded as follows. When the fertility and viability of $F_{1}$ hybrids is good, a comprehensive genetic analysis is possible. As a disadvantage 
there is not much to be analyzed. However, we believe that we may find such incompatibilities between the genetic components, which are not expressed in $F_{1}$, but in later generations. For example, incompatibility between an alien $\mathrm{X}$ chromosome and a homozygous autosome is not visible in $F_{1}$. Furthermore, it is reasonable to believe that genetic incompatibilities leading to postzygotic isolation accumulate with time, also after the completion of species formation. In the hybrid, they all interact, and details of the syndrome become more and more difficult to grasp when the degree of isolation, i.e., the number of incompatible interacting systems increases. It is to be expected that the genetically controlled traits which express themselves as disadvantageous (subvitality, lethality, sterility) in hybrids, have some positive role in pure species. When the disadvantage is weak, it is perhaps possible to find out the normal function of the respective genes.

Among the virilis species group, the hybrids of $D$. virilis and $D$. lummei are the most viable and fertile of all. Yet, their relatedness is not so close. The phylogenetic tree of the virilis group has been constructed on the basis of salivary gland chromosomes (Throckmorton, 1982) and confirmed by the biochemical techniques (MacIntyre amd Collier, 1986; Coyne and Orr, 1989a). In the tree, the diversification of $D$. virilis from the common ancestor of $D$. americana, $D$. novamexicana and $D$. lummei is ancient. The other two species have developed considerable postzygotic isolation towards $D$. virilis. What is different in $D$. lummei?

\section{MATERIAL AND METHODS}

\section{Fly stocks and crosses}

We used in most experiments Drosophila virilis marker stocks $126(b ; g p ; c d ; p e)$ and MM (Majors Marked, $w ; b ; g p ; c d ; p e)$. The stock 126 is from The Institute of Developmental Biology, USSR Academy of Sciences, Moscow. Stock NEW also contains $b ; g p ; c d ; p e$. It is actually 126 refreshed through crossing with wild type 1422 . MM was made through introduction of $w$ from a stock $B x w$ into 126. Also $139(y a p)$ was used in one experiment.

The recombination map positions of markers used in this work are as follows: yellow 1-3, apricot 1-136, white, 1-105, broken 2-188, gapped 3-118, cardinal 4-32, and peach 5-203 (Alexander, 1976). All large chromosomes are thus recessively marked. Dot chromosome is very small, comprising $0 \cdot 1$ per cent of the length of the recombination map. D. virilis stock 1422 from Groeningen, The Netherlands was used as a wild type, as well as stock Batumi $\boldsymbol{A}$ from Batumi, Georgia, U.S.S.R.

To represent Drosophila lummei, we used stock number $1101 \mathrm{~S}$, wild type, originating from Overkalix, northern Sweden, and stock number 1143, also wild type, from Hokkaido, Japan. Two other stocks, 1100 (Kuopio, Finland) and luJapFu (Hokkaido, Japan) served as donors of one $\mathrm{X}$ chromosomal inversion.

First generation hybrids were obtained reciprocally between $126, \mathrm{MM}$, and $1101 \mathrm{~S}$. To produce novel combinations of the genetic elements, $F_{1}$ males were crossed back to females of marker strain $D$. virilis. This always produced 16 combinations of heterozygous or homozygous autosomes, together with sex chromosome set depending on the direction of cross in $\mathrm{P}$ generation. Through repeated backcrossing to $\mathrm{MM}$ stock, the inversion $\operatorname{In}(1) a+b$ from $D$. lummei was introduced into $D$ virilis chromosome. The inversion contains wild type allele of white, and it was maintained in heterozygous condition in females. Such heterozygous females were crossed with $F_{1}$ males to construct flies having a part of $\mathrm{X}$ chromosome from $D$. lummei, and autosomes either heterozygous, or homozygous for $D$. virilis. The role of $\mathrm{X}$ chromosome was further studied through crossing the $y$ $a p$ stock with $D$. lummei, and backcrossing the $\mathrm{F}_{1}$ females to both parental species.

\section{Survival from egg to adult}

To measure the survival of parental stocks and hybrids, two females and four males were put in plastic vials on $5 \mathrm{ml}$ of malt medium (Lakovaara, 1969). They were allowed to lay eggs 24 hours at $25^{\circ} \mathrm{C}$. The eggs laid were counted. We did not make any estimates of the proportion of unfertilized eggs. The vials with developing larvae were kept at $17^{\circ} \mathrm{C}$ or $25^{\circ} \mathrm{C}$, and the emerging adults were counted. When the eclosion had been ceased for two days, the number of pupal cases was counted. Mouldy, bacterially infected, or dried tubes were discarded.

\section{Statistical methods}

The contribution of chromosomes and their interactions to the survival of different phenotypes in backcross generations were calculated through a modification of analysis of factorial experiments (Snedecor and Cochran, 1967). The grouping of data for the analysis is presented in table 1 , because it may help to understand figs 2 and 4 in the Results. 
Table 1 Grouping of phenotype $N$ values used to sum up the factorial effect totals, for the main effects and interactions between the chromosomes (expanded from Snedecor and Cochran, 1967; 6th edn, p. 360). $N$ values are summed into groups a and b, and the factorial effect total (=difference between means) is $\Sigma_{a}-\Sigma_{b}$. Statistical tests are conducted between groups a and b. If the sex is added, the table would be four times as large

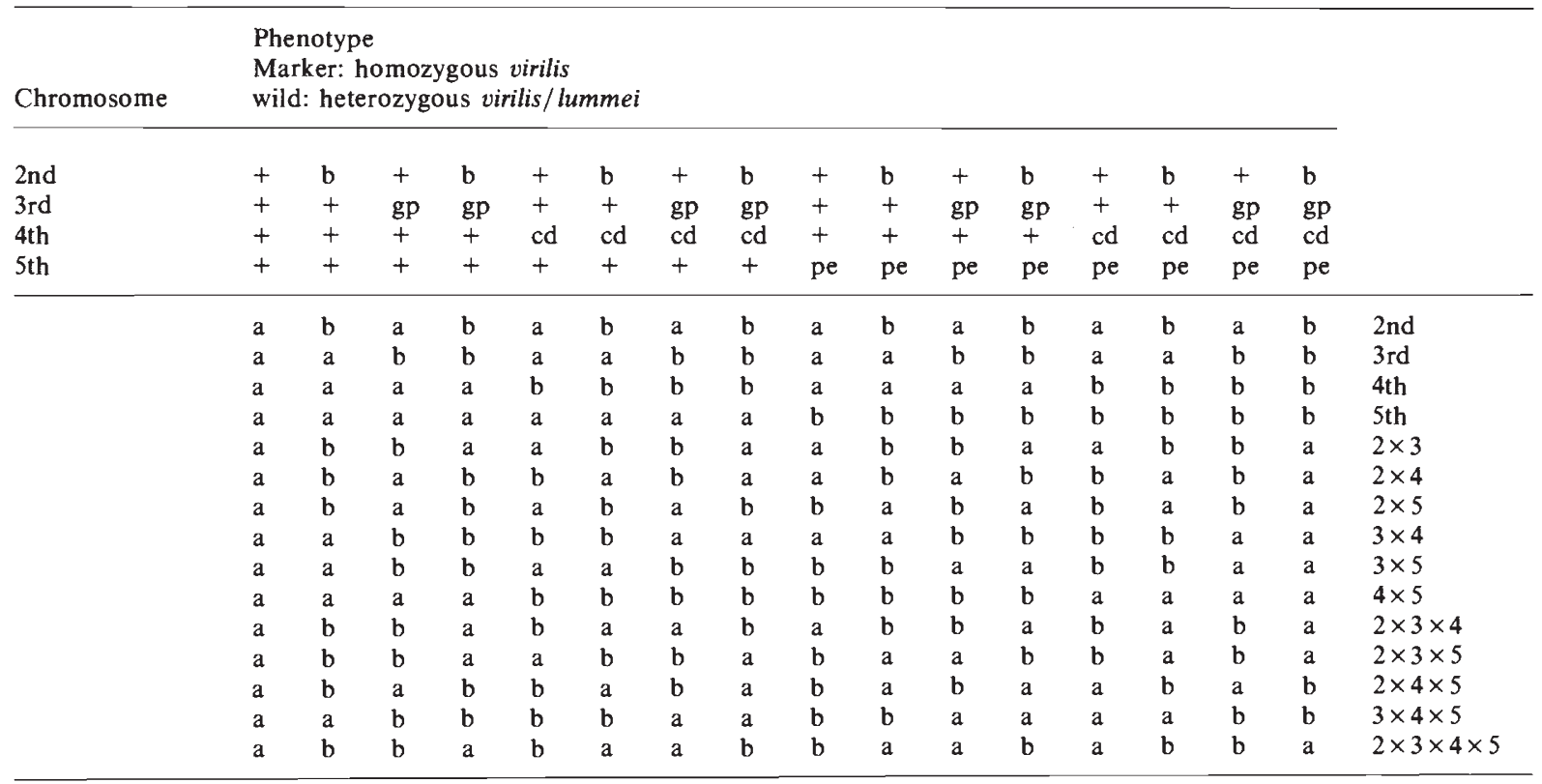

As a statistical test, we have used $G$-test for comparing observed and expected segregation ratios.

\section{RESULTS}

\section{Survival from exg to pupariation and to adult in $P$ and $F_{1}$}

In average, the mortality of the eggs of $D$. virilis at $25^{\circ} \mathrm{C}$ before pupariation was $22 \cdot 8$ per cent $(N=$ 3047 ), and the mortality within pupal case was $5 \cdot 7$ per cent $(N=2351)$. At $17^{\circ} \mathrm{C}$, the mortality values were 30.8 per cent $(N=1928)$ and 5.8 per cent $(N=1335)$, respectively. Table 2 displays in detail the results of all stocks used, as percentages of eggs surviving at both steps of development. The weakest stock was 126, which is old, inbred marker stock. Best was MM, which has recently gone through crossing with wild type and new purification of markers.

In D. lummei, 38.0 per cent of the 573 counted eggs kept at $25^{\circ} \mathrm{C}$ died before pupariation, and 8.5 per cent of pupae failed to emerge. At $17^{\circ} \mathrm{C}, D$. lummei survived better during early development: 23.4 per cent of 474 eggs died before pupariation, but 22.9 per cent of pupae died before emerging to adults.
The total fitness of reciprocal hybrids was about the same, and much lower than in parental generation. Reciprocal hybrids show clearly different developmental profiles. From $D$. virilis female $\times D$. lummei male hybrids (data at $25^{\circ} \mathrm{C}$, excluding cross $\mathrm{NEW} \times 1143,3864$ eggs), $47 \cdot 0$ per cent died before pupariation, and 24.9 per cent of pupae failed to emerge. At $17^{\circ} \mathrm{C}$, the mortality values were 50.0 per cent $(N=3476)$, and $17 \cdot 4$ per cent $(N=1739)$. In the reciprocal cross, $D$. lummei females $\times D$. virilis males, a slightly larger proportion of the progeny died (66.8 per cent, 1587 eggs), but of them, 65.0 per cent of 1587 eggs failed to pupariate, and only 5.0 per cent of pupae died before adult emergence. The values at $17^{\circ} \mathrm{C}$ are very similar: $66 \cdot 2(N=1845)$ and $7 \cdot 2$ per cent $(N=624)$.

The hybrids of stocks NEW of $D$. virilis and 1143 of $D$. lummei differed clearly from the others. Survival of eggs mothered by $D$. virilis was much better. Their mortality within pupal case was especially low $(5 \cdot 3$ per cent, $N=414)$. [This is correlated with the low proportion of developmental disorders among these hybrids. Only 0.8 per cent of them suffer from eye syndrome (Heikkinen and Lumme, in preparation), which occurred in other hybrid progenies of $D$. virilis at a frequency of almost 10 per cent.] On the other hand, the 
Table 2 Number of eggs counted, and proportion (percentage) of them surviving until pupariation (P) and adult eclosion (A), in two rearing temperatures

\begin{tabular}{|c|c|c|c|c|c|c|}
\hline \multirow[b]{3}{*}{ Genotype } & \multicolumn{6}{|c|}{ Temperature } \\
\hline & \multicolumn{3}{|l|}{$25^{\circ} \mathrm{C}$} & \multicolumn{3}{|l|}{$17^{\circ} \mathrm{C}$} \\
\hline & Eggs & $\mathrm{P}$ & $\mathbf{A}$ & Eggs & $\mathrm{P}$ & A \\
\hline virilis 1422 & 116 & $71 \cdot 6$ & $69 \cdot 8$ & 80 & $56 \cdot 3$ & $53 \cdot 8$ \\
\hline virilis 126 & 358 & $61 \cdot 7$ & $57 \cdot 3$ & 167 & $69: 5$ & $66 \cdot 5$ \\
\hline virilis $\mathbf{M M}$ & 273 & 93.4 & $86 \cdot 8$ & 190 & $72 \cdot 6$ & $61 \cdot 6$ \\
\hline lummei $1101 \mathrm{~S}$ & 454 & $65 \cdot 0$ & $56 \cdot 2$ & 471 & $76 \cdot 6$ & $59 \cdot 1$ \\
\hline lummei 1143 & 119 & $58 \cdot 8$ & $58 \cdot 8$ & - & & \\
\hline $126 \times 1422$ & 2300 & $77 \cdot 9$ & $73 \cdot 6$ & 1491 & $69 \cdot 5$ & $66 \cdot 1$ \\
\hline $126 \times 1101 S$ & 1589 & $47 \cdot 2$ & $36 \cdot 9$ & 1521 & $38: 1$ & $34 \cdot 3$ \\
\hline $1101 \mathrm{~S} \times 126$ & 857 & $34 \cdot 2$ & $31 \cdot 9$ & 1155 & $32 \cdot 2$ & $30 \cdot 2$ \\
\hline $\mathrm{MM} \times 1101 \mathrm{~S}$ & 2275 & $57 \cdot 0$ & $41 \cdot 9$ & 1955 & $59 \cdot 3$ & $46 \cdot 8$ \\
\hline $1101 \mathrm{~S} \times \mathrm{MM}$ & 730 & $35 \cdot 9$ & $34 \cdot 8$ & 690 & $36 \cdot 5$ & $33 \cdot 3$ \\
\hline $\mathrm{NEW} \times 1143$ & 649 & $63 \cdot 8$ & $60 \cdot 4$ & - & & \\
\hline $1143 \times \mathrm{NEW}$ & 116 & $4 \cdot 2$ & $3 \cdot 0$ & - & & \\
\hline
\end{tabular}

survival of $1143 \times \mathrm{NEW}$ eggs was much worse than that of other hybrids (table 3 ). This observation was made after completing most of the experiments described here, and this special case will be analyzed later in detail.

The sex ratios of the survived hybrid adults are presented in table 3 . In $D$. virilis $\times D$. lummei progenies grown at $25^{\circ} \mathrm{C}$, there were significantly fewer males than females, in both replicates. At $17^{\circ} \mathrm{C}$, also the control cross between $D$. virilis stocks produced less males.

\section{Segregating generations}

In the following, we compare the viabilities of different chromosomal combinations obtained through crossing the $\mathrm{F}_{1}$ males to marker stock $D$. virilis. Only relative viabilities can be measured, since the markers used can be scored only in adult flies.
In fig. 1 we present the proportions of males and females in pooled data comprising of three different types of crossing, including the control cross between marker stock and wild type $D$. virilis. All crosses produce 16 autosomal combinations, with various combinations of sex chromosomes. The sex chromosome set of course depends on the direction of cross made in the parental generation. The statistical analysis of the results is depicted in fig. 2.

\section{Control virilis $\times$ (virilis $\times$ virilis)}

Fig. 1 displays combined results from several crosses, using as marker stocks either 126 or NEW, and as the wild type, 1422 or $A$. There was no significant inhomogeneity among the results.

The total deviation from expected phenotype frequencies is small, hardly significant $\left(G_{(31)}=\right.$ $48.45, P<0.05)$. The sex ratio is even: 1890 males

Table 3 Sex ratios of the control cross and $F_{1}$ hybrids at two different temperatures

\begin{tabular}{|c|c|c|c|c|c|c|}
\hline \multirow[b]{3}{*}{ Genotype } & \multicolumn{6}{|c|}{ Temperature } \\
\hline & \multicolumn{3}{|l|}{$25^{\circ} \mathrm{C}$} & \multicolumn{3}{|l|}{$17^{\circ} \mathrm{C}$} \\
\hline & Males & Females & $G_{(1)}$ & Males & Females & $G_{(1)}$ \\
\hline $126 \times 1422$ & 841 & 851 & 0.059 & 457 & 529 & $5 \cdot 262^{*}$ \\
\hline $126 \times 1101 S$ & 246 & 340 & $15 \cdot 144^{* * *}$ & 229 & 292 & $7.637^{* *}$ \\
\hline $1101 S \times 126$ & 150 & 123 & 2.675 & 163 & 186 & $1 \cdot 517$ \\
\hline $\mathrm{MM} \times 1101 \mathrm{~S}$ & 418 & 536 & $14 \cdot 633^{* * *}$ & 421 & 493 & $5.677^{*}$ \\
\hline $1101 \mathrm{~S} \times \mathrm{MM}$ & 119 & 135 & 1.009 & 120 & 110 & 0.435 \\
\hline
\end{tabular}

\footnotetext{
$* P<0.05$.

** $P<0.01$.

*** $P<0 \cdot 001$.
} 
Phenotype

$+;+;++$

b; $+;+;+$

tigp;t;+

b; gp; ; +

$t: t ; c d ;+$

b;t;cd;t

tigp;cd;t

b; gp;cd;+

$+;+;+; p e$

b; ; + ; pe

tigp;t;pe

b; gp; t;pe

$+;+$; cd;pe

b;t;cd;pe

t;gp;cd;pe

b; gp; cd; pe
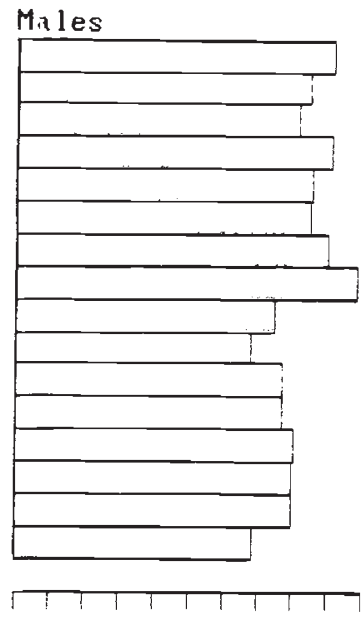

Phenotype

$+;+;++$

bit; $+;+$

$+;$ gp;t;+

b;gp;t;+

t; icdit

b; ; cd; +

tigp;cd;t

b;gp;cdit

$+;+;+$ pe

b; $;+;$ pe

tigp;tipe

b;gp;t;pe

titicdipe

biticd;pe

tigp;cdipe

b;gp;cd;pe
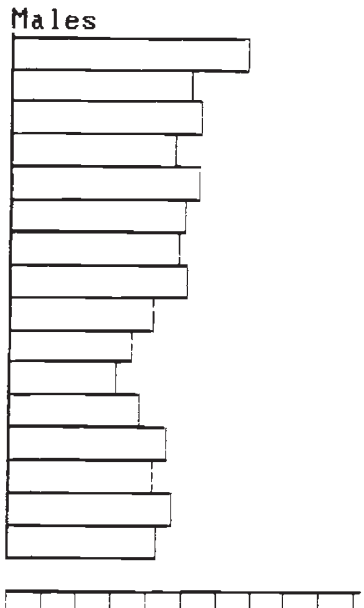

Phenotype

$+;+;++$

b;t;+;t

tigpit;t

bigp; ;+

t; t;cd; +

biticd; +

tigp;cd;+

bigp;cd; +

$t ;+;+; \mathrm{pe}$

bit;+;pe

tigp;t;pe

b;gp;+;pe

tit;cd;pe

biticdipe

tigp;cd;pe

bigp;cdipe
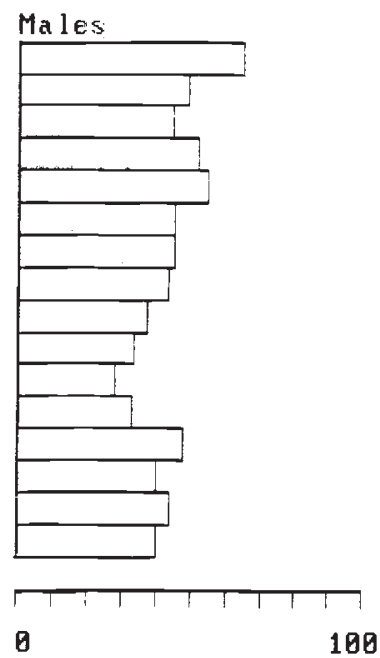

Frequency $(\%)$ as proportion of the most frequent

Figure 1 Relative frequencies of phenotypes from control and hybrid backcrosses. $D$. virilis marker stock was $b$; $g p ; c d$; pe. $D$. lummei and $D$. virilis in controls were of wild type. Marker phenotype then indicates homozygous $D$. virilis chromosome; + is for heterozygous. Among each progeny, the most frequent phenotype (in hybrids, $+;+;+;+$ female) is set to have the column of 100 units.

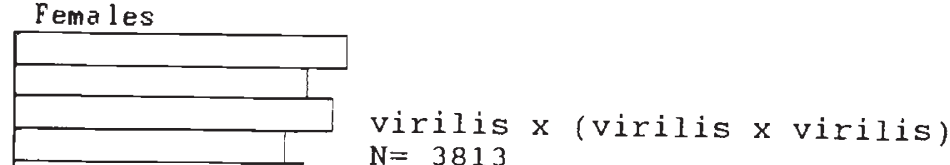

Fema les
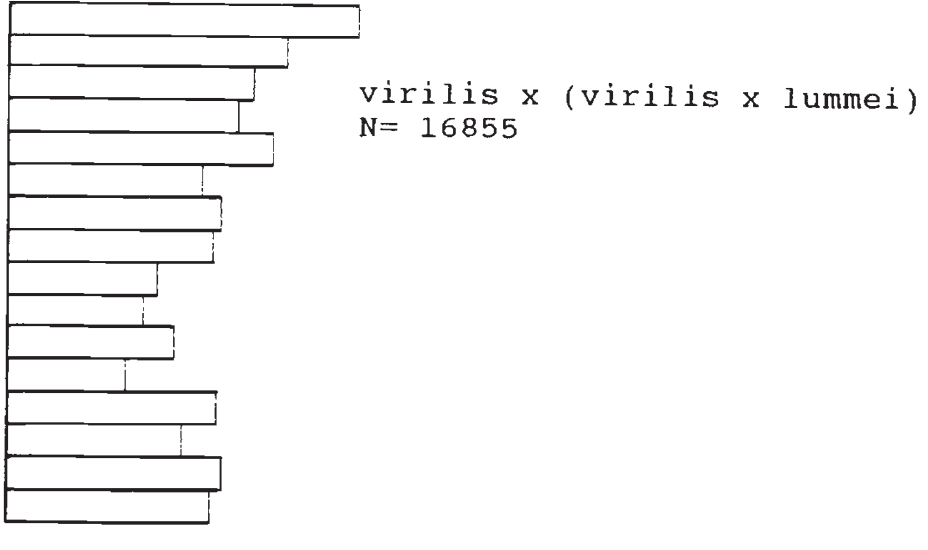

Females

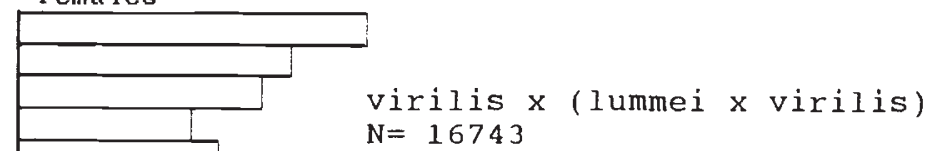

$N=16743$ 
$\begin{array}{lll}1 & 2 & 3\end{array}$

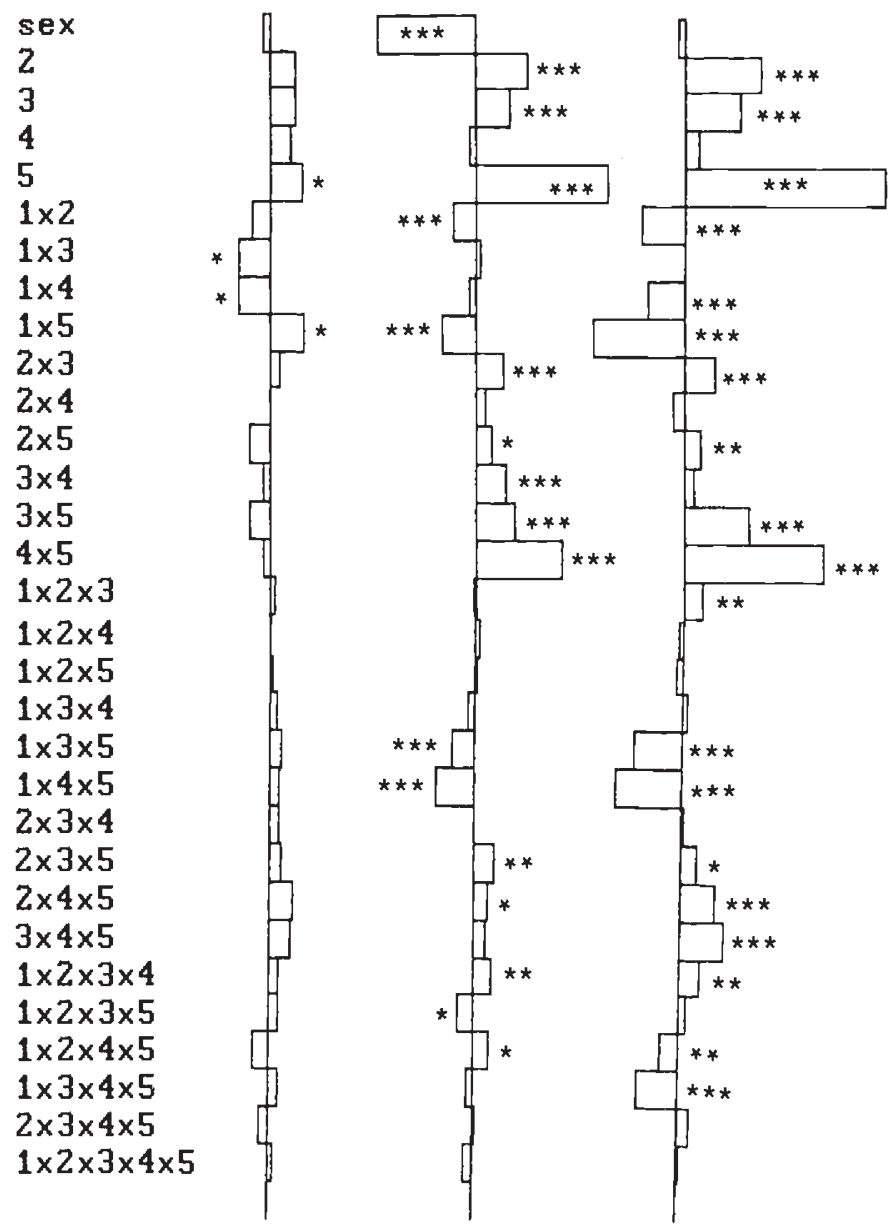

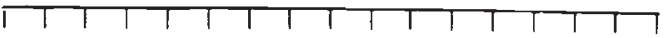

\section{Relative contribution of chromosomes \& interactions}

Figure 2 Main effects and interactions of genetic elements (Analysis of data in fig. 1). The contributions are scaled to be proportional to the expected number $[(\Sigma \mathrm{a}-\Sigma \mathrm{b}) /(N / 2)]$ (See table 1.). One division $=0.1$. 1. Control virilis $\times($ virilis $\times$ virilis $)$, 2. virilis $\times$ (virilis $\times$ lummei), 3. virilis $\times$ (lummei $\times$ virilis). The significance was tested by $G$-test $\left({ }^{*} P<0.05 ;{ }^{* *} P<0.01,{ }^{* * *} P<0.001\right)$.

and 1923 females were counted $\left(N_{\mathrm{m}} / N_{\mathrm{f}}=\right.$ $0.983)$.

In the total material, there were $1980 \mathrm{pe} /+$ heterozygotes and $1833 \mathrm{pe} / \mathrm{pe}$ homozygotes. This difference is to be understood as the main effect of the fifth chromosome showing subviability of mutant homozygotes. Deviation from the expected $1: 1$ segregation is significant $\left(G_{(1)}=5 \cdot 214, P<\right.$ $0 \cdot 05)$. Interactions $1 \times 3,1 \times 4$, and $1 \times 5$, where 1 means the sex, are significant at the 5 per cent level, $G_{(1)}$-values being from $5 \cdot 3$ to $5 \cdot 7$. This is due to the small differences in the frequencies of the corresponding phenotypes among males and females.

\section{Heterospecific virilis $\times$ (virilis $\times$ lummei)}

The total data depicted in fig. 1 contains altogether 16845 individuals, collected from different combinations of stocks during diverse experiments. As $D$. virilis, both 126 and NEW have been used, and as D. lummei, wild type stocks $1101 \mathrm{~S}$ and 1143 . 
No inhomogeneity was observed, and therefore we present the pooled results pooled over all replicates.

Males from this backcross carry heterospecific sex chromosome combination $\mathrm{X}^{\mathrm{vi}} / \mathrm{Y}^{1 \mathrm{u}}$, as in the $F_{1}$ with more aberrant sex ratio, $D$. virilis $\times D$. lummei. Females carry homozygous combination $\mathrm{X}^{\mathrm{vi}} / \mathrm{X}^{\mathrm{vi}}$, which is fittest together with all-heterozygous autosomes in phenotype $(+;+;+;+)$.

Among the backcross progeny, the sex ratio was significantly aberrant $\left(N_{\mathrm{m}} / N_{\mathrm{f}}=0 \cdot 788, N=\right.$ $\left.16,845, G_{(1)}=236, P \ll 0.001\right)$. This is expressed in fig. 2 as the large negative main effect of "sex". The weighted mean sex ratio in $\mathrm{F}_{1}(D$. virilis $\times D$. lummei) (table 3 ) was very closely the same, $0 \cdot 791$ $\left(N=2975, G_{(1)}=40.57, P<0.001\right)$.

The frequencies of the different autosomal combinations deviate significantly from the expected. All 16 classes should be equally frequent. Among females, the deviations are wider than among the males, but the patterns resemble each other. The main effects of the autosomes 2, 3, and 5 are highly significant, and in all cases, there are more heterozygous than homozygous flies in the progeny. The interactions $1 \times 2$ and $1 \times 5$ are highly significant, which means that the relative shortage of $b / b$ and pe/pe homozygotes is much worse among the females. This is not due to $\mathrm{X}$-autosome incompatibility, since both $\mathrm{X}$ chromosomes of the females arised from $D$. virilis.

Between the autosomes, the interactions $2 \times 3$, $3 \times 4,3 \times 5$, and $4 \times 5$ are significant at the level of $P<0.001$, and all of them have positive sign (fig. 2 , table 4). This means that chromosomes of similar origin are functioning better together. As a consequence, the phenotype $b ; g p ; c d$; pe is not the weakest one, even if it contains all the homozygous large autosomes. The relative fitnesses of autosomal combinations similar/different origin are calculated in table 4 .

To make this point clear, we display in table 5 the observed relative fitnesses of each autosomal phenotype combination in females. The data is pooled over both backcrosses. The phenotype frequencies were transformed to relative fitness by dividing the number of each class by the class containing most of the flies (invariably, the heterozygous or double heterozygous phenotype).

\section{Heterospecific virilis $\times$ (lumme $\times$ virilis)}

As can be seen in figs 1 and 2, the relative proportions of different phenotypes follow the same pattern as in the previous cross, but the variation of the viabilities among phenotypes was clearly amplified, especially among the females. Here the males carry homospecific set of sex chromosomes, $\mathrm{X}^{\mathrm{vi}} / \mathrm{Y}^{\mathrm{vi}}$. Females are heterozygous $\mathrm{X}^{\mathrm{vi}} / \mathrm{X}^{1 \mathrm{u}}$. The overall sex ratio is close to unity $\left(N_{\mathrm{m}} / N_{\mathrm{f}}=0.984\right.$, $\left.N=16,743, G_{(1)}=1 \cdot 1, P \gg 0 \cdot 1\right)$.

The deviation of female phenotype frequencies from the expected was wider than in the previous cross, where the females were homozygous $\mathrm{X}^{\mathrm{vi}} / \mathrm{X}^{\mathrm{vi}}$. The variability in viabilities of the various autosomal combinations is due to some second degree $\mathrm{X} /$ autosome/autosome-interaction, which can be expressed by saying that the negative interaction of heterozygous to homozygous autosomes is

Table 4 Relative fitness (w) of flies homozygous for each autosome in backcrosses (for heterozygotes $w=1 \cdot 0$ ), or flies carrying one pair homozygous, other pair heterozygous autosomes (in flies having both homozygous + both heterozygous, $w=1 \cdot 0$ ). Data is pooled over replicates, different stocks, and sexes

\begin{tabular}{llll}
\hline \multirow{2}{*}{$\begin{array}{l}\text { Chromosome } \\
\text { vir } \times(\text { vir } \times \text { lum })\end{array}$} & $\begin{array}{l}\text { vir } \times(\text { lum } \times \text { vir }) \\
N=16855\end{array}$ & $\begin{array}{l}\text { Total } \\
N=33625\end{array}$ \\
\hline 2 & $0.880^{* * *}$ & $0.829^{* * *}$ & $0.833^{* * *}$ \\
3 & $0.917^{* * *}$ & $0.873^{* * *}$ & $0.896^{* * *}$ \\
4 & 1.015 & $0.964^{*}$ & 0.991 \\
5 & $0.719^{* * *}$ & $0.607^{* * *}$ & $0.660^{* * *}$ \\
Interaction & & & \\
$2 \times 3$ & $0.935^{* * *}$ & $0.929^{* * *}$ & $0.931^{* * *}$ \\
$2 \times 4$ & 0.980 & 1.026 & 1.001 \\
$2 \times 5$ & $0.961^{*}$ & $0.958^{* *}$ & $0.961^{* * *}$ \\
$3 \times 4$ & $0.927^{* * *}$ & 0.974 & $0.948^{* * *}$ \\
$3 \times 5$ & $0.906^{* * *}$ & $0.852^{* * *}$ & $0.880^{* * *}$ \\
$4 \times 5$ & $0.808^{* * *}$ & $0.707^{* * *}$ & $0.758^{* * *}$ \\
\hline
\end{tabular}

\footnotetext{
${ }^{*} P<0.05$.

** $P<0.01$.

*** $P<0.001$, tested with $G_{(1)}$.
} 
Table 5 Relative fitness (w) of homozygous or heterozygous autosomes 2 to 5 , and their pairwise combinations in female progenies in virilis $\times($ virilis $\times$ lummei $)$ and virilis $\times$ (lummei $\times$ virilis) backcrosses $(N=17895)$. For testing the interaction, double homozygotes and double heterozygotes were pooled to have $w=1.0$ and compared with sum of $A^{\mathrm{vi} / \mathrm{vi}} B^{\mathrm{vi} / \mathrm{lu}}$ and $\mathrm{A}^{\mathrm{vi} / \mathrm{lu}} \mathrm{B}^{\mathrm{vi} / \mathrm{vi}}$

\begin{tabular}{|c|c|c|c|c|c|c|}
\hline A & B & $A^{\mathrm{vi} / \mathrm{vi}} \mathbf{B}^{\mathrm{vi} / \mathrm{vi}}$ & $A^{v i / v i} B^{v i / l u}$ & $A^{v i / l u} B^{v i / v i}$ & $A^{v i / l u} B^{v i / l u}$ & $\mathrm{~A} / \mathrm{B}$ \\
\hline 2 & & $0.803^{* * *}$ & & & $1 \cdot 000$ & \\
\hline 3 & & $0.887 * * *$ & & & $1 \cdot 000$ & \\
\hline 4 & & $0.946^{* * *}$ & & & $1 \cdot 000$ & \\
\hline 5 & & $0.581 * * *$ & & & $1 \cdot 000$ & \\
\hline 2 & 3 & 0.717 & 0.778 & 0.860 & $1 \cdot 000$ & $0 \cdot 954^{* *}$ \\
\hline 2 & 4 & 0.759 & 0.806 & 0.948 & 1.000 & 0.997 \\
\hline 2 & 5 & 0.464 & 0.813 & 0.589 & 1.000 & $0.957^{* *}$ \\
\hline 3 & 4 & 0.843 & 0.841 & 0.899 & $1 \cdot 000$ & $0.944 * * *$ \\
\hline 3 & 5 & $0 \cdot 544$ & 0.775 & 0.487 & 1.000 & $0.801^{* * *}$ \\
\hline 4 & 5 & 0.605 & 0.706 & 0.386 & $1 \cdot 000$ & $0.681^{* * *}$ \\
\hline
\end{tabular}

${ }^{*} P<0.05$

** $P<0.01$.

*** $P<0.001 ; G_{(1)}$ test.

amplified in the presence of $\mathrm{X}^{1 \mathrm{u}}$, or because of the heterozygosity of $\mathrm{X}$.

\section{Recombinant $X$-chromosome: Inversion substitution lines}

D. lummei has in $\mathrm{X}$ chromosome a fixed double inversion $\operatorname{In}(1) a+b$ in comparison to $\mathrm{X}$ of $D$. virilis. We constructed inversion substitution lines, originally to obtain $D$. virilis with photoperiodic diapause. For this purpose, MM females $(w ; b ; g p ; c d ; p e)$ were crossed with single $D$. lummei males. $\mathrm{F}_{1}$ daughters were then backcrossed to MM males. From the next generation, $w /+$ females were backcrossed to MM males, and this backcrossing was continued. During the first few generations, the autosomal markers $b, g p, c d$ and $p e$ were picked to be homozygous. After 50 generations of backcrossing, the lines were maintained by $w /+\times w$ crossings within the line. An attempt was made to make the lines homozygous for $w^{+}$. allele (and $\operatorname{In}(1) a+b$ ), but in only two of the four lines this succeeded, because of the high degree of sterility of the $w^{+}$-hemizygous males (Heikkinen and Lumme, in preparation).

In table 6, we present the phenotype frequencies in four inversion substitution lines. What is relevant for the present topic is that the viability of $w^{+}$males was strongly reduced. Summed over lines and generations from table $6,44 \cdot 3$ per cent of females were heterozygous. It deviates significantly from the expected $1: 1$ segregation $(N=$ $\left.3874, G_{(1)}=50 \cdot 08, P<0.001\right)$. In males, the deviation is very much larger. Only 12.6 per cent were hemizygous $w^{+}\left(N=2577, G_{(1)}=1706, P \ll 0.001\right)$.
Table 6 Phenotype frequencies in inversion substitution lines. The backcrossing of heterozygous $w /+$ females to MM w males was continued until $\mathrm{BC}_{50}$. After this, the lines were maintained through $w /+\times w$ crosses within the line. Total sums of phenotypes are: $w / w 2157, w /+1717, w / Y 2301$, $+/ \mathrm{Y} 276$

\begin{tabular}{llrrr}
\hline & & \multicolumn{3}{c}{ Time of inspection } \\
\cline { 4 - 5 } $\begin{array}{l}\text { Origin of } \\
\text { In }(1) a+b\end{array}$ & Phenotype & $\mathrm{BC}_{9-10}$ & $\mathrm{BC}_{50}$ & $G_{10}$ \\
\hline \multirow{2}{*}{$1101^{*}$} & $w / w$ & 148 & 102 & 208 \\
& $w /+$ & 138 & 58 & 151 \\
& $w$ & 221 & 114 & 252 \\
\multirow{4}{*}{1101} & + & 34 & 9 & 28 \\
HETEX & $w / w$ & 265 & 81 & 175 \\
& $w /+$ & 276 & 49 & 164 \\
\multirow{3}{*}{$l u J a p F u$} & $w$ & 280 & 92 & 175 \\
& + & 46 & 7 & 23 \\
& $w / w$ & 251 & 110 & 270 \\
& $w /+$ & 219 & 53 & 165 \\
1100 & $w$ & 266 & 107 & 223 \\
& + & 26 & 3 & 39 \\
& $w / w$ & 192 & 107 & 248 \\
& $w /+$ & 141 & 113 & 190 \\
& $w$ & 193 & 109 & 242 \\
& + & 10 & 27 & 24 \\
\hline
\end{tabular}

The total sex ratio is aberrant because of the missing $w^{+}$males $\left(N_{\mathrm{m}} / N_{\mathrm{f}}=0.665, G_{(1)}=262, P \ll\right.$ $0 \cdot 001)$. This indicates that hemizygous $\operatorname{In}(1) a+b$ from $D$. lummei causes poor survival when implemented into $D$. virilis genome.

The sex ratio in the two pure breeding $\operatorname{In}(1) a+$ $b$ substitution lines (females homozygous) remained uneven in the first few generations after making them homozygous (1101*: $N_{\mathrm{m}} / N_{\mathrm{f}}=$ $\left.\left.32 / 189=0 \cdot 169, \quad G_{(1)}=124, \quad P \ll 0 \cdot 001\right)\right) ; \quad 1100$ : $\left.N_{\mathrm{m}} / N_{\mathrm{f}}=76 / 257=0.296, \quad G_{(1)}=104, \quad P \ll 0.001\right)$. 
This demonstrates that the observed hybrid weakness is a male sex-limited character. Meiotic drive as an explanation for the under-representation of the $\operatorname{In}(1) a+b$ males is also excluded. The genetic basis was analysed as follows.

\section{Heterospecific recombinant $X$ in combination with alien autosomes}

Heterozygous $w /+$ females from inversions substitution line 1101 (HETEX) were crossed with $F_{1}$ $(1101 \mathrm{~S} \times \mathrm{MM})$ and $\mathrm{F}_{1}(\mathrm{MM} \times 1101 \mathrm{~S})$ males. The autosomal phenotype frequencies of the resulting male progenies are displayed in fig. 3 .

For the analysis of the data, males from both crosses, which had different $Y$ chromosome but showed similar autosomal phenotype frequencies, were pooled together. Fig. 4 displays the contribution of each homozygous $D$. virilis autosome to the weak viability of carriers of $\operatorname{In}(1) a+b$. The contribution of fifth chromosome is twice as large as the contribution of the second chromosome. The interaction of the fifth and second does not deviate significantly from additive, but it is closer

\section{Phenotype Males}

tit;t;t

b; $;$; ; t

t;gp;t;+

b; gs; ; ;

titicdit

b; ticd; +

tigp;cd;t

bigp;cd;t

$t ; t ; t ; p e$

b;t;t;pe

$t ;$; $;+;$ pe

b; gp;t;pe

titicd;pe

b;t;cd;pe

tigp;cdipe

b;gp;cd;pe
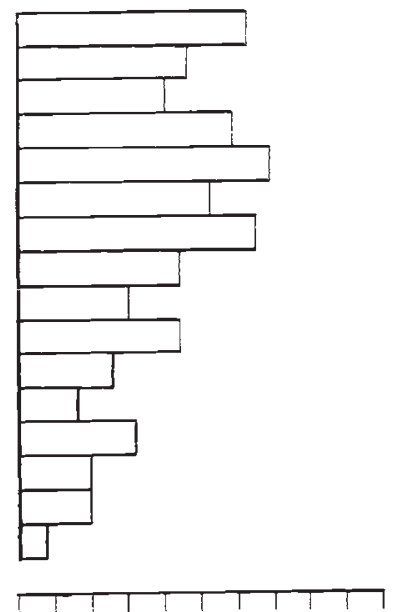

Phenotype

$+;+;+;+$

b;t;t;t

$t$;gp;t;+

b; gp; ; ;

t;icd;t

b; ; icd;+

tigp;cdit

bigp;cd; +

$t ;+; t ; p e$

b;t; $;$ pe

t;gp;t;pe

b; gp;t;pe

t; t;cdipe

b;t;cd;pe

tigp;cd;pe

b;gp;cd;pe
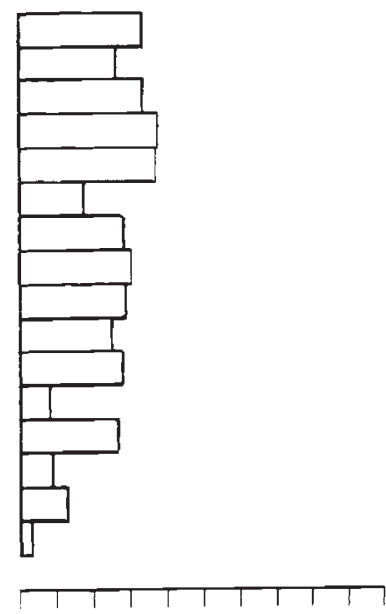

$B$

Frequency (\%) as proportion of the most frequent
Females

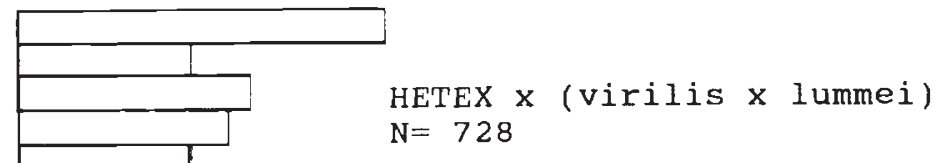

Figure 3 Relative frequencies of autosomal combinations in males and females carrying In(1) $a+b$ from crosses HETEX $\times$ $(\mathrm{MM} \times 1101 \mathrm{~S})$ and HETEX $\times(1101 \mathrm{~S} \times \mathrm{MM})$. In the first cross, white-eyed flies were discarded. In the latter cross, females are overrepresented, because $w /+$ and $+/+$ genotypes are not separable, and white-eyed males are not included. The markers in the stocks are: HETEX: In (1) $a+b w^{+} /$Standard $w ; b ; g p ; c d ; p e . \mathrm{MM}: w ; b ; g p ; c d ; p e .1101 \mathrm{~S} D$. lummei $+;+;+;+$. 


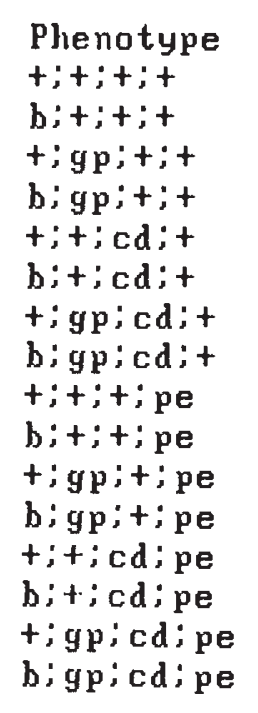

\section{Males}
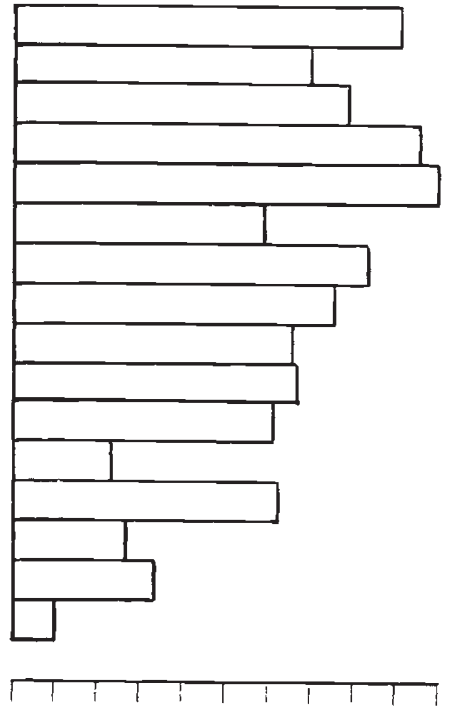

B

100

Frequency $(\%)$ as proportion of the most frequent
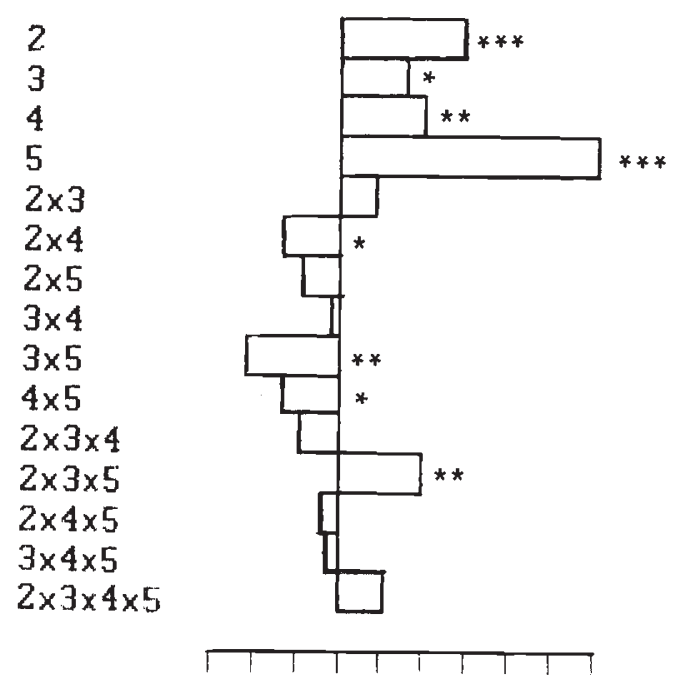

\section{Relative contribution of chromosomes \& interactions}

Figure 4 (Upper panel) Relative frequencies of autosomal combinations in males carrying hemizygous $\operatorname{In}(1) a+b(\mathrm{~N}=902)$ from crosses depicted in fig. 3. (Lower panel) Main effects and interactions of homozygous $D$. virilis autosomes causing the weak viability of carriers of $\operatorname{In}(1) a+b$. The contributions are scaled to be proportional to the expected number $[(\Sigma \mathrm{a}-\Sigma \mathrm{b}) /(N / 2)]$ (See table 1.). One division $=0 \cdot 1 .\left({ }^{*} P<0.05,{ }^{* *} P<0.01, * * * P<0.001, G\right.$-test $)$.

to multiplicative model. When the fitness of $+/+$ is set to 1.000 , the relative fitnesses of other combinations of the second and fifth chromosomes are for $b ;+0.849,+$; pe 0.629 , and $b$; pe 0.358 . The multiplicative prediction for double homozygote is 0.534 . It is to be expected that some of the autosome-autosome interactions detected even irrespective of the origin of intact $\mathrm{X}$ chromosome, 
are acting here, too, but they cannot override the negative interaction of $\operatorname{In}(1) a+b$ and autosomes 2 and 5 . Here $b$; pe phenotype survives badly, even if both autosomes are from $D$. virilis.

\section{Recombination within the $X$ chromosome}

In the previous experiment, only a part of the $\mathrm{X}$ implemented into the genome of $D$. virilis was from D. lummei. Because there exists a possibility that the hybrid weakness is not caused solely by the incompatibility between hemizygous $\operatorname{In}(1) a+b$ and homozygous $D$. virilis autosomes, but by the incompatibility between distal and proximal parts of the $\mathrm{X}$ itself, the following cross was made. $\mathrm{F}_{1}$ females from a cross $y a p \times$ wild-type $D$. lummei were backcrossed to $y$ ap or to $D$. lummei. The allele yellow marks the distal tip of the $\mathrm{X}$ chromosome, and apricot is a marker of the part of the chromosome included in the $\operatorname{In}(1) a+b$. The frequencies of phenotypes in $\mathrm{BC}_{1}$ are presented in table 7. The overall recombination frequency of the markers is 0.489 , indicating undisturbed crossing over outside of the inversion.

From the inspection of the results in table 7 it can be seen that none of the novel male combinations from backcrosses to $D$. virilis has much reduced viability. The reason is that the proportion of autosomes homozygous for $D$. virilis is still rather low in $\mathrm{BC}_{1}$. Among the males (pooled over both stocks), the relative fitness of $a p^{+}$phenotypes is $w=0.808\left(G_{(1)}=12.4, P<0.001\right)$, and that of $y^{+}, w=0.896\left(G_{(1)}=3 \cdot 3, P>0.05\right)$, but the recombination within $\mathrm{X}$ seems not to be harmful. Thus, the subvitality of $\operatorname{In}(1) a+b$ in males having otherwise $D$. virilis background is caused by its incompatibility with several homozygous autosomes.

In table 7 we also present results of backcrossing $\mathrm{F}_{1}$ to D. lummei. When crossed with stock $1101 \mathrm{~S}$, the virilis segment of $\mathrm{X}$ including the standard gene arrangement for $\operatorname{In}(1) a+b$ has a clear disadvantage with partially homozygous lummei autosomes. Additional reduction of fitness is achieved when the distal tip of the $\mathrm{X}$ also is from $D$. virilis, but the tip alone seems not to be incompatible. In cross involving Japanese stock 1143 the standard $\mathrm{X}$ of $D$. virilis is not subvital. This difference between the D. lummei stocks is autosomal, and will be analysed later.

\section{DISCUSSION}

Our studies about adaptive and non-adaptive genetic differences between Drosophila virilis and $D$. lummei have been mainly directed to traits other than viability. The data presented here have been collected when genetically analysing other traits which are or will be reported elsewhere (cold shock resistance: Heino and Lumme, 1989; male and female sterility, developmental disorders, and mate choice, Heikkinen and Lumme, in preparation).

The major findings of the present analysis can be summarized as follows.

$F_{1}$ was clearly weaker than parental stocks: fewer eggs survived until adults. In data pooled over all experiments, the relative fitnesses (w) were as follows. Out of $D$. virilis eggs, $69 \cdot 8$ per cent

Table 7 Phenotype frequencies in progenies from crosses between $D$. virilis 139 y ap and two wild type (++) stocks of $D$. lummei, 1143 and $1101 \mathrm{~S}$

\begin{tabular}{|c|c|c|c|c|c|c|c|c|}
\hline \multirow[b]{3}{*}{ Phenotype } & \multicolumn{4}{|c|}{$(y a p \times++) \times y a p$} & \multicolumn{4}{|c|}{$(y a p \times++) \times++$} \\
\hline & \multicolumn{2}{|l|}{1143} & \multicolumn{2}{|l|}{$1101 \mathrm{~S}$} & \multicolumn{2}{|l|}{1143} & \multicolumn{2}{|l|}{$1101 \mathrm{~S}$} \\
\hline & Female & Male & Female & Male & Female & Male & Female & Male \\
\hline++ & 148 & 115 & 119 & 142 & 160 & 26 & 560 & 150 \\
\hline$y+$ & 105 & 112 & 108 & 118 & & 39 & & 148 \\
\hline$+a p$ & 115 & 125 & 146 & 133 & & 40 & & 91 \\
\hline y ap & 99 & 144 & 145 & 201 & & 33 & & 53 \\
\hline Sum & 467 & 496 & 518 & 594 & 160 & 138 & 560 & 442 \\
\hline Sex ratio & $1 \cdot 062$ & & $1 \cdot 147$ & & 0.863 & & 0.789 & \\
\hline$G_{(1)}$ & 0.9 & & $5 \cdot 2^{*}$ & & $1 \cdot 6$ & & $13 \cdot 9^{* * *}$ & \\
\hline $\begin{array}{l}\text { Phenotype ratio } \\
G_{(3)}\end{array}$ & $11 \cdot 8^{* *}$ & $4 \cdot 9$ & $8 \cdot 5^{*}$ & $25 \cdot 4^{* * *}$ & & $3 \cdot 8$ & & $65 \cdot 0^{* * *}$ \\
\hline
\end{tabular}

\footnotetext{
$* P<0.05$.

$* * P<0.01$.

*** $P<0.001$.
} 
survived to adults $(\mathrm{w}=1)$. Survival of $D$. lummei eggs was $57 \cdot 7$ per cent $(\mathrm{w}=0 \cdot 826), D$. virilis $\times D$. lummei hybrids 40.5 per cent $(\mathrm{w}=0.581)$, and of $D$. lummei $\times D$. virilis hybrids $32 \cdot 2$ per cent $(\mathrm{w}=$ $0 \cdot 462$ ). Altogether, 4008 adults emerged from the 5903 counted eggs of pure species, $(w=1)$, and 4081 adults from 10772 eggs from hybridizations $(\mathrm{w}=0.558)$. We do not know how large a proportion of the eggs was unfertilized.

Reciprocal hybrid progenies have different sex ratio. $D$. virilis mothers had the sex ratio in their progeny: $N_{\mathrm{m}} / N_{\mathrm{f}}=0.791 \quad(N=2975), D$. lummei mothers had as many sons as daughters $\left(N_{\mathrm{m}} / N_{\mathrm{f}}=\right.$ $0.996, N=1106$ ).

Reciprocal hybrids with D. lummei stock $1101 \mathrm{~S}$ differed more with respect to the developmental profile of the lethality than of the total numbers of surviving eggs. The hybrids mothered by $D$. virilis survived slightly better, but a large proportion of their mortality occurred during adult metamorphosis within pupal case. Probably correlated with this, the emerging adults frequently suffered from visible developmental disorders in eyes, antennae, wings, and abdominal chitinization (Heikkinen and Lumme, in preparation). Many of them could survive only in the sheltered situation in laboratory. The Japanese stock 1143 was different from Swedish stock 1101S, in pupal mortality, and also in frequency of developmental disorders.

The $D$. virilis $\times D$. lummei hybrids (especially males) are also more susceptible to cold shock than pure species or the progeny of the reciprocal cross (Heino and Lumme, 1989).

In backcross generations, the second, third and fifth chromosomes expressed heterosis. In all possible sex chromosome backgrounds, the relative viability of flies heterozygous for a given autosome was higher than that of flies homozygous for $D$. virilis autosome. This is not to be expected on the basis of comparing $P$ and $F_{1}$ generations: flies having all autosomes as heterozygous (reciprocal $F_{1}$ values) were clearly less fit than parental species, when measured by egg to adult survival.

The role of maternal cytoplasm cannot explain this apparent controversy between first and second hybrid generation, because mothers of both generations were pure species. A similar unresolved situation was observed in a study of cold shock resistance (Heino and Lumme, 1989). There, the $F_{1}$ was weaker than the most similar backcross phenotype. The role of elimination of the tiny sixth chromosome was speculated (about the elimination, see Sokolov, 1948, 1959; Mitrofanov and Sidorova, 1979).
Any two of the autosomes work relatively better when they are identical, i.e., both heterozygous, or both homozygous. Out of six possible combinations, $2 \times 4$ is the only exception (tables 4 and 5). Fitnesses of double homozygotes follow well estimates made according to multiplicative interaction, but genotypes with one chromosome homozygous and one heterozygous fall below this prediction. This is a very interesting finding. While in the species pair studied, the degree of postzygotic isolation is rather weak, polygenic accumulation of small effects in all chromosomes seems to be going on. Interestingly enough, this effect was much stronger in females than in males.

Inversion $\operatorname{In}(1) a+b$ from $D$. lummei is incompatible with the homozygous autosomes of $D$. virilis, causing strongly decreased viability of carrier males. All D. virilis autosomes take part in this incompatibility system, the role of fifth being strongest. Reciprocally, the standard X of $D$. virilis is incompatible with the homozygous autosomes of D. lummei (this interaction was, however, straindependent and thus polymorphic in D. lummei). The participating autosomes are not yet analyzed because of the lack of suitable markers in $D$. lummei.

Our results are not contradictory with the "two rules of speciation" (Coyne and Orr, 1989b), even if the isolation is weakly expressed in $F_{1}$. In this work, we investigated a quantitative and relative subvitality among hybrids. The few analyzed cases in the literature concern rather absolute inviability of males of females. The rules hold for quantitative viability, too.

Haldane's rule (1922) states that the heterogametic sex is affected first. In our case, the weak incompatibilities between heterozygous and homozygous autosomes were more pronounced among the females. This seems to be contradictory with Haldane's rule, but this effect was really rather weak. With this same pair of species, Mitrofanov and Sidorova (1981) demonstrated another autosomal lethal interaction, affecting only females. Certain backcross phenotypes gave no female progeny, when crossed again with $D$. lummei males.

In our results, the stronger male sex-limited viability interactions obeyed Haldane's rule. Even if we have been able to construct pure breeding $D$. virilis carrying $\operatorname{In}(1) a+b$ of $D$. lummei, only hemizygous males are subvital, not the homozygous females.

Coyne's rule says that $X$ chromosome is responsible for the earliest and strongest postzygotic isolation. This was confirmed here, too. However, our analysis also reveals the elements 
interacting with the $\mathrm{X}$. In our case, proximal half of $\mathrm{X}$ included in the inversion $\operatorname{In}(1) a+b$ of $D$. lummei is subvital with homozygous alien $(D$. virilis) autosomes, especially the fifth. Reciprocally, standard arrangement of the same part of X is subvital with combination of $D$. lummei autosomes (which of them, is to be analysed). The observed polymorphism in response of autosomes of $D$. lummei will be of experimental value in analysing this incompatibility.

Acknowledgements This work was supported by The Finnish Academy (Project 1051049). We thank Dr M. Ashburner for informing us about the results on $\mathrm{Hmr}$ gene prior to publication.

\section{REFERENCES}

ALEXANDER, M. L. 1976. The genetics of Drosophila virilis. In Ashburner, M. and Novitski, E. (eds) The Genetics and Biology of Drosophila, vol 1c, Academic Press, London, pp. 1365-1427.

COYNE, J. A. AND ORR, H. A. 1989a. Patterns of speciation in Drosophila. Evolution, 48, 362-381.

COYNE, J. A. AND ORR, H. A. 1989 b. Two rules of speciation. In Otte, D. and Endler, J. A. (eds) Speciation and its Consequences, Sinauer Associates, Sunderland, Mass, pp. 180-207.

HALDANE, J. B. S. 1922. Sex ratio and unisexual sterility in hybrid animals. J. Genetics, 12, 101-109.

HEINO, R. AND LUMME, J. 1989. Inheritance of cold shock tolerance in hybrids of Drosophila virilis and Drosophila lummei. Genetica, 79, 17-25.

HUTTER, P. AND ASHBURNER, M. 1987. Genetic rescue of inviable hybrids between Drosophila melanogaster and its sibling species. Nature, 327, 331-333.

HUTTER, P., ROOTE, J. AND ASHBURNER, M. (1990) A genetic basis for the inviability of hybrids between sibling species of Drosophila. Genetics, 124, 909-920.

LAKOVAARA, S. 1969. Malt as a culture medium for Drosophila species. Drosoph. Inf. Serv., 44, 128.
LUMME, J. AND KERANEN, L. 1978. Photoperiodic diapause in Drosophila lummei Hackman is controlled by an Xchromosomal factor. Hereditas, 89, 261-262.

MACINTYRE, R. J. AND COLliER, G. E. 1986. Protein evolution in the genus Drosophila. In Ashburner, M., Carson, H. L. and Thompson, J. N. Jr. (eds) The Genetics and Biology of Drosophila, vol 3e, Academic Press, London, pp. 39-146.

MITROFANOV, v. G. AND SIDOROVA, N. V. 1979. Phenogenetic analysis of chromosome behavior during mitosis in female Drosophila virilis $\times$ male Drosophila lummei hybrids. Genetika Moscow), 15, 1221-1227. In Russian with an abstract in English.

MITROFANOV, V. G. AND SIDOROVA, N. V. 1981 Genetics of the sex ratio anomaly in Drosophila hybrids of the virilis group. Theor. Appl. Genet., 59, 17-22.

PATTERSON, J. T. AND GRIFFEN, A. B. (1944) A genetic mechanism underlying species isolation. Univ. Texas Publ. 4415.

PATTERSON, J. T. AND STONE, W. S. 1952. Evolution in the Genus Drosophila. The Macmillan Co., New York.

SNEDECOR, G. W. AND COCHRAN, W. G. 1967. Statistical Methods. The Iowa State University Press, Ames, Iowa.

SOKOLOv, N. N. 1948. * Eliminatsija hromosom u mesvidovih gibridov drosofili i problemi otdalennoi gibridisatsii. Dokl. Akad. Nauk SSSR, 59, 163-166.

SOKolov, N. N. 1959. $\dagger$ Vsaimodeistvie jadra i tsitoplasmi pri otdalennoi gibridisatsii sivotnih. Isdatelstvo Akademii Nauk SSSR, Moscow.

THROCKMORTON, L. H. 1982. The virilis species group. In Ashburner, M., Carson, H. L. and Thompson, J. N. Jr. (eds) The Genetics and Biology of Drosophila, vol 3b, Academic Press, London, pp. 227-296.

WATANABE, T. K. 1979. A gene that rescues the lethal hybrids between Drosophila melanogaster and D. simulans. Japan J. Genetics, 54, 325-331.

* Н. Н. СОКОЛОВ ЭЛИМИНАЦИЯ ХРОМОСОМ У МЕЖВИДОВЫХ ГИБРИДОВ ДРОЗОФИЛЫ И ЛРОЬЛЕМЫ ОТДАЛЕННОЙ ГИБРИДИЗАЦИИ Доклады Академии Наук СCCP 1948. Tом LIX, No. 1.

$\dagger$. Н. СОКОЛОВ ВЗАИМОДЕЙСТВИЕ ЯДРА И ЦИТОПААЗМЫ ПРИ ОТДААЕННОЙ ГИБРИДИЗАЦИИ ЖИВОТНЫХ. издательство академии наук ссср Москва1959. 